\title{
Genome-wide association study to identify genetic variants present in Japanese patients harboring intracranial aneurysms
}

\author{
Koichi Akiyama ${ }^{1}$, Akira Narita ${ }^{1}$, Hirofumi Nakaoka ${ }^{1}$, Tailin Cui ${ }^{1}$, Tomoko Takahashi ${ }^{1}$, Katsuhito Yasuno ${ }^{1}$, \\ Atsushi Tajima ${ }^{1}$, Boris Krischek ${ }^{2}$, Ken Yamamoto ${ }^{3}$, Hidetoshi Kasuya ${ }^{4}$, Akira Hata ${ }^{5}$ and Ituro Inoue ${ }^{1}$
}

An intracranial aneurysm (IA), which results in a subarachnoid hemorrhage with a high mortality on rupture, is a major public health concern. To identify genetic susceptibility loci for IA, we carried out a multistage association study using genome-wide single nucleotide polymorphisms (SNPs) in Japanese case-control subjects. In this study, we assessed evidence for association in standard approaches, and additional tests with adjusting sex effects that act between genetic effect and disease. Consequently, five SNPs $\left(P=1.31 \times 10^{-5}\right.$ for rs 1930095 of intergenic region; $P=1.32 \times 10^{-5}$ for rs4628172 of TMEM195; $P=2.78 \times 10^{-5}$ for rs7781293 of TMEM195; $P=4.93 \times 10^{-5}$ for rs7550260 of $A R H G E F 11$; and $P=3.63 \times 10^{-5}$ for rs9864101 of IQSEC1) with probabilities of being false positives $<0.5$ were associated with IA in Japanese population, and the susceptibility genes could have a role in actin remodeling in the ELNILIMK pathway. This study indicates the presence of several susceptibility loci that deserve further investigation in the Japanese population.

Journal of Human Genetics (2010) 55, 656-661; doi:10.1038/jhg.2010.82; published online 8 July 2010

Keywords: cerebrovascular disease; genetics; genome-wide association study; intracranial aneurysm; sex effect; single nucleotide polymorphism; subarachnoid hemorrhage

\section{INTRODUCTION}

Intracranial aneurysm (IA) (MIM105800) is a cerebrovascular disease and has a reported prevalence of 3-6\%. As the rupture of an IA leads to subarachnoid hemorrhage (SAH), which often results in death or severe physical disability, the disease is considered to be a major public health concern. ${ }^{1-3}$ Much of the etiology of IA still remains unknown, but subjects with a family history of IA have a higher risk of being affected by IA, suggesting that some genetic components contribute to predisposition to developing an IA. Previously, we conducted a genome-wide linkage study in affected Japanese sib pairs ${ }^{4}$ and showed through subsequent association studies that the single nucleotide polymorphisms (SNPs) of ELN and LIMK1 on chromosome 7 was significantly associated with IA. ${ }^{5}$ We also discovered one SNP of LOXL2 on chromosome 8 associated with IA, and a possible genegene interaction of LOXL2 with ELN/LIMK1. ${ }^{6}$ Other loci of the linkage analyses in the Japanese population include $14 \mathrm{q} 23$, replicated by an association study. ${ }^{7}$ In other ethnic groups, the perlecan gene (HSPG2) at $1 \mathrm{p} 36.1-36.4,{ }^{8}$ the versican gene (CSPG2) at $5 \mathrm{q} 14.3^{9}$ and Kallikrein at $19 \mathrm{q} 13^{10}$ have been proposed as susceptibility genes for IA by case-control association study, among others.
Genome-wide association studies (GWASs) on complex diseases using high-density SNP arrays have recently been exemplified by a number of groups. ${ }^{11,12}$ In a previous study, a multicenter collaboration regarding IA genetics led to complete a multistage GWAS that identified common variants that contribute to IA formation in three large cohorts: a Finnish cohort of 920 cases and 985 controls, a Dutch cohort of 781 cases and 6424 controls and a Japanese cohort of 495 cases and 676 controls. $^{11}$ Consequently, common SNPs with odds ratios (ORs) of 1.22-1.36 on chromosomes $2 \mathrm{q}, 8 \mathrm{q}$ and $9 \mathrm{p}$ were identified and showed significant association with IA. Because the three loci account only for a small fraction of the genetic variance underlying IA, it remains a challenge to identify another genetic variant such as common variants with population-specific impacts on the risk of IA.

Intracranial aneurysm is most likely to have a multifactorial etiology, involving complex interactions of genetic and environmental risk factors. Smoking habits, hypertension, frequent alcohol intake and female gender are known risk factors for aneurysm formation and subsequent SAH. ${ }^{13-16}$ There is a possibility that these nongenetic risk factors might hinder the identification of genetic effects. Therefore, in the case of evaluating genuine genetic effects of SNPs contributing to

\footnotetext{
${ }^{1}$ Department of Molecular Life Sciences, Basic Medical Science and Molecular Medicine, Tokai University School of Medicine, Kanagawa, Japan; ${ }^{2}$ Department of Neurosurgery, University of Tübingen, Tübingen, Germany; ${ }^{3}$ Division of Molecular Population Genetics, Department of Molecular Genetics, Medical Institute of Bioregulation, Kyushu University, Fukuoka, Japan; ${ }^{4}$ Department of Neurosurgery, Neurological Institute, Tokyo Women's Medical University, Tokyo, Japan and ${ }^{5}$ Department of Public Health, Graduate School of Medicine, Chiba University, Chiba, Japan

Correspondence: Dr I Inoue, Department of Molecular Life Sciences, Basic Medical Science and Molecular Medicine, Tokai University School of Medicine, 143 Shimokasuya, Isehara, Kanagawa 259-1193, Japan.

E-mail: ituro@is.icc.u-tokai.ac.jp
}

Received 23 February 2010; revised 30 May 2010; accepted 5 June 2010; published online 8 July 2010 
IA, we first dealt with the interaction of SNPs with the invariable risk factor, female gender, at a genome-wide level, rather than those with other modifiable lifestyle factors such as smoking habits.

To gain a more comprehensive understanding of IA pathogenesis, we carried out a multistage association study using genome-wide SNPs in Japanese case-control data sets. For this purpose, we conducted a genome-wide assessment of SNP-gender interactions by statistically examining whether gender could affect an association of each SNP with IA. After controlling for the gender-effect, we identified five promising candidate loci associated with IA, by classifying and applying the best-suited tests for SNPs.

\section{MATERIALS AND METHODS}

\section{Study design}

We first performed a two-stage GWAS in Japanese IA case-control cohorts. To perform the most powerful and cost-effective study, the top-ranked $1 \%$ of the genotyped SNPs of each GWA stage were used for the following stage $\left(1 \% \times 1 \%=0.01 \%\right.$ of tagged autosomal SNPs). The statistical power, $1-\beta_{\mathrm{GWAS}}$, is estimated to be more than $85 \%$ for the two-stage association study, assuming that $\alpha_{\mathrm{GWAS}}$ is $2 \times 10^{-7}$, the prevalence of IA is $5 \%$ in the Japanese population, genotypic relative risk is 1.6 and allele frequency of the polymorphism is 0.45 , calculated by the CaTS Power Calculator for two-stage association studies, available on the web site of Center of Statistical Genetics, University of Michigan (Supplementary Figure 1). ${ }^{17}$ Additional statistical tests to examine possible confounding between SNP and gender, and effect modification of the genetic effect by gender were performed (the details are shown below). For the analysis of SNPs modified by gender, we also calculated statistical powers specific for male and female cohorts in the GWAS. These powers $\left(1-\beta_{\text {male }}\right.$ and $\left.1-\beta_{\text {female }}\right)$ are estimated to be more than 18 and $22 \%$, respectively, under the same conditions in which $1-\beta_{\mathrm{GWAS}}$ was estimated.

In the final intensified study, we estimated false-positive report probabilities (FPRPs) of associations of the SNPs selected carefully from the GWAS stage by using all available case-control samples including GWAS cohorts to examine which SNPs would be noteworthy to be reported.

\section{Subjects}

The Ethics Committees of Tokai University, Tokyo Women's Medical University and Chiba University approved the study protocols, and all the participants gave written informed consent. We recruited a total of 1069 IA patients and 904 controls from the three university hospitals described above and their affiliated hospitals. The 495 cases $(48.9 \%)$ and 676 controls $(74.8 \%)$ have been also used in the previous GWAS by Bilguvar et al. ${ }^{11}$ Of the cases, $27.7 \%$ had familial history of IA, and SAH was observed in 727 cases (68.0\%). The female to male ratio of cases and controls were 1.56 and 0.62 , respectively. The presence of IA was confirmed by conventional angiography, three-dimensional computed tomography angiography, magnetic resonance angiography or surgical findings, when applicative. Controls were recruited at the time of periodic health examination or from outpatients with conditions other than IAs and/or SAHs, such as idiopathic headache. About $92 \%$ of them were examined by magnetic resonance excluding the presence of IA. All the remaining control subjects had no medical and familial history of SAH. Adding them to the control group did not influence genetic frequency in this study (data not shown). The information for lifestyles and hypertension was obtained by a standard questionnaire, and each question was asked and confirmed by a skilled research coordinator and medical doctor (summarized in Table 1). Genomic DNA was extracted from peripheral blood or saliva according to the standard protocol.

\section{Exclusion criteria for subjects}

In the first stage of the GWAS, 499 subjects (300 patients/199 controls) were genotyped, 920 (460/460) were added to the second stage and the remaining $534(310 / 224)$ were additionally genotyped in the intensified study. We excluded subjects in whom the genotyping call rate was $<0.95$ and 0.7 in first and second stages of GWAS, respectively.

In the first stage of GWAS, we also excluded subjects who genetically deviated from most other subjects on the basis of the result of multidimensional
Table 1 The detailed numbers of the subjects used in the study

\begin{tabular}{|c|c|c|c|}
\hline & Non-IA & $I A$ & Total \\
\hline \multicolumn{4}{|c|}{ Starting cohort size } \\
\hline Total & 904 & 1069 & 1973 \\
\hline Male & 551 & 408 & 959 \\
\hline Female & 353 & 661 & 1014 \\
\hline \multicolumn{4}{|c|}{ Partitioning of samples } \\
\hline \multicolumn{4}{|c|}{ GWAS (first stage) } \\
\hline Total & 199 & 300 & 499 \\
\hline Male & 115 & 112 & 227 \\
\hline Female & 84 & 188 & 272 \\
\hline \multicolumn{4}{|c|}{$\begin{array}{l}\text { GWAS (newly added in } \\
\text { second stage) }\end{array}$} \\
\hline Total & 460 & 460 & 920 \\
\hline Male & 280 & 177 & 457 \\
\hline Female & 180 & 283 & 463 \\
\hline \multicolumn{4}{|c|}{ GWAS (second stage) } \\
\hline Total & 659 & 760 & 1419 \\
\hline Male & 395 & 289 & 684 \\
\hline Female & 264 & 471 & 735 \\
\hline \multicolumn{4}{|c|}{ Intensive study } \\
\hline Total & 882 & 1069 & 1951 \\
\hline Male & 537 & 408 & 945 \\
\hline Female & 345 & 661 & 1006 \\
\hline
\end{tabular}

Final cohort (quality controlled)

GWAS (first stage)

Total

Male

194

111

Female

83

108

482

219

GWAS (newly added in

second stage)

455

Male

276

Female

179

GWAS (second stage)

Total

649

Male

387

Female

262

Intensified study

Total

Male

Female

515

338

Mean age ( \pm s.d.)

$\mathrm{SAH}+(\%)$

Hypertension ${ }^{\mathrm{a}, \mathrm{b}}$

Yes

No

$2( \pm 14.0)$

-

346

355

180

263

Smoking habit ${ }^{\mathrm{a}}$

Ever

Never

370

Alcohol drinking habit ${ }^{a}$

Ever

326

452

907

171

507

281

399

1389

740

726

279

662

461

1880

906

974

391

636

$59.1( \pm 13.3)$

Never

217

-

67.8

505

342

851

697

Abbreviations: GWAS, genome-wide association study; IA, intracranial aneurysm; SAH, subarachnoid hemorrhage.

aDifferences between total and analyzed sample size were caused by incomplete clinical information.

bHypertension was defined as systolic blood pressure $>140 \mathrm{~mm} \mathrm{Hg}$ and/or diastolic blood pressure $>90 \mathrm{~mm} \mathrm{Hg}$. 
scaling analysis, which was performed to identify genetic outliers. Furthermore, to detect cryptic relatedness (undiscovered kinship among subjects), probabilities of identity-by-state were estimated for each pair of subjects, and the data sets were reduced so that there was no substantial genetic kinship within four degrees among the remaining subjects. The calculation of identity-by-state probabilities and multidimensional scaling analysis were carried out using the software package PLINK. ${ }^{18}$

In the intensified study, we also excluded subjects with a genotyping call rate $<0.8$

\section{Genotyping and exclusion criteria for SNPs}

In the first stage of the GWAS, genotyping was carried out using the HumanHap300 or HumanHap300-Duo Genotyping BeadChips (Illumina, San Diego, CA, USA), and a total of 312712 SNPs were tested in both BeadChips. The SNPs were then filtered on the basis of genotyping call rate ( $>95.0 \%$ in either two types of BeadChips), minor allele frequency $(>0.02)$ and deviation from Hardy-Weinberg equilibrium $\left(P<1.0 \times 10^{-5}\right)$. The X-linked SNPs were also excluded.

In the second stage, the top-ranked $1 \%$ of all the SNPs analyzed in the first stage and the newly added 920 subjects were genotyped using the GoldenGate genotyping assay (Illumina). These 2304 SNPs were also quality controlled on the basis of genotyping call rate $(>90.0 \%)$, minor allele frequency $(>0.01)$ and the Hardy-Weinberg equilibrium $\left(P<1.0 \times 10^{-4}\right)$. To confirm the concordance of the genotype data obtained on different platforms, 20 of the subjects ( 10 patients/10 controls) included in the first stage were regenotyped as well.

In the intensified study, the remaining 532 subjects and all the other subjects studied in the previous two stages were genotyped for the 22 SNPs whose $P$-values ranked $1 \%$ of all those analyzed in the second stage using the TaqMan SNP Genotyping Assays (Applied Biosystems, Foster City, CA, USA). We confirmed the reliability of the genotype data by comparing the genotypes of the subjects studied in the previous two stages with those obtained by the HumanHap300/HumanHap300-Duo Genotyping BeadChips or the GoldenGate genotyping assay. The genotype concordance rate of the TaqMan data in the intensified study with the HumanHap data in the first GWAS stage was $99.4 \% \pm 0.5$ (mean \pm s.d.), and that with the GoldenGate data in the second GWAS stage was $98.9 \% \pm 1.1$

\section{Statistical analysis}

To test for association of each SNP with IA, we assumed an additive (in logodds scale) model. The sex-specific effect modification can be characterized by the large difference of ORs between males and females. We estimated sexspecific OR by using a logistic regression model. Under the null hypothesis of no effect modification by sex, the statistic $\psi$, the logarithm of the ratio of these sex-specific ORs, follows asymptotically normal distribution with variance $\sigma^{2}$, the sum of the variances of sex-specific ORs. ${ }^{19}$

$$
\psi=\log \mathrm{OR}_{\text {male }} / \mathrm{OR}_{\text {female }} \approx N\left(0, \sigma^{2}\right) \text { (average } \sigma^{2}=0.06 \text { at the GWAS stage) }
$$

If the test $P$-value was $<0.2$, (corresponding to $\exp (\psi)=\mathrm{OR}_{\text {male }} / \mathrm{OR}_{\text {female }}>1.37$ or $<0.73$ under the above average $\sigma^{2}$ ), which might be large or small enough to consider that the SNP-effect differed between males and females, we stratified our data by sex, tested using each stratum, and defined the SNPs as 'class M'.

We supposed that gender was a confounding factor for association between the SNP and IA if the absolute value of the log-ratio of OR adjusted by gender to crude OR is $>0.1$ (roughly $>10 \%$ difference of ORs), and defined as 'class C. The adjusted and crude ORs were calculated using the logistic regression models with and without gender as a covariate, respectively.

For SNPs that were neither modified nor confounded by gender, defined as 'class N', the Cochran-Armitage (CA) trend test (one degree of freedom) and general genotypic test (two degrees of freedom) were performed. In contrast to the SNPs that were modified by gender, those tests were separately performed in male and female subjects (class M). For the SNPs that were confounded by gender, the Mantel extension test (one degree of freedom), and the generalized Cochran-Mantel-Haenszel test (two degrees of freedom) were performed (class C).

We also examined the validity of the assumption of additivity (in log-odds scale) in the association tests by comparing the logistic regression models with and without dominance effect.
To explore the likelihood that the results represent true associations, we computed the FPRP under different assumptions. ${ }^{20}$ The previous probability of a true association was set between 0.01 and 0.0001 , as it is regarded as an adequate level for genome-wide scan. ${ }^{21}$ We considered that the SNP might be noteworthy when the FPRP was below 0.5 for suggestive association or 0.2 for significant association..$^{20,22}$

\section{RESULTS}

\section{Quality control}

At the first stage of the GWAS, genome-wide genotyping in 499 Japanese subjects was performed, and we extracted 250507 of 312712 SNPs through the rigorous filtering described above. According to multidimensional scaling analysis based on identity-by-state distance (Supplementary Figure 2), 12 genetic outliers and 3 related individuals were excluded from the association analysis. Two subjects with low genotyping call rate were further excluded, and samples obtained from a total of 482 subjects were finally used in the first stage study. The detailed numbers of the subjects after filtering through the GWAS stage and the intensified study were shown in Table 1 .

\section{Genome-wide case-control association study}

In the first stage of the GWAS, 250507 filtered autosomal SNPs were analyzed in 482 subjects. In the following stage, 1833 filtered SNPs were analyzed in a cohort of 1389 subjects, which included 482 subjects studied in the first stage and 907 who were newly added. The summary of statistical analysis in the first stage is available on the genome-wide association database (https://gwas.lifesciencedb.jp/ cgi-bin/gwasdb/gwas_study.cgi?id=cerebral).

We investigated genetic effects of the filtered 250507 autosomal SNPs in the first stage, of which 50740 (20.2\%) were modified by gender (class $\mathrm{M}$ ). We then applied the CA trend test and the 2 d.f. genetic test to each of the sex-stratified data sets (Table 2). Meanwhile, 32413 SNPs (13.0\%) were confounded by gender (class C). We estimated the association by Mantel extension test and generalized Cochran-Mantel-Haenszel test. For the remaining 167354 (66.8\%), the CA trend test and the 2 d.f. genetic test were applied to the crude data set (class $\mathrm{N}$ ). We arranged the $P$-values from all tests in ascending order and recruited the top 2304 SNPs, corresponding to $0.92 \%$ of the filtered SNPs, to the second stage. Of the 2304 SNPs, 1043 were from class M, 205 from class $C$ and 1056 from class N. By this screening, we could select out SNPs whose $P$-values were $<0.008$.

In the second stage, 920 samples (460 controls/460 cases) were genotyped by GoldenGate assay for SNPs that had passed the first

Table 2 SNP classification according to possible effect modification and confounding by sex

\begin{tabular}{|c|c|c|c|c|}
\hline & $\begin{array}{l}\text { Sex effect } \\
\text { modification } \\
\text { (class } M \text { ) }\end{array}$ & $\begin{array}{c}\text { Sex } \\
\text { confounding } \\
\text { (class } C \text { ) }\end{array}$ & $\begin{array}{c}\text { Others } \\
\text { (class N) }\end{array}$ & Total \\
\hline Passed SNPs in first stage & 50740 & $\begin{array}{c}32413 \\
\downarrow 0.92 \% \\
(P<0.008)^{\mathrm{a}}\end{array}$ & 167354 & 250507 \\
\hline Genotyped in second stage & 1043 & $\begin{array}{c}205 \\
\downarrow \text { SNP quality } \\
\text { control }\end{array}$ & 1056 & 2304 \\
\hline Passed SNPs in second stage & 838 & $\begin{array}{c}162 \\
\downarrow 0.95 \% \\
\left(P<2 \times 10^{-4}\right)^{\mathrm{a}}\end{array}$ & 833 & 1833 \\
\hline Intensified study & 7 & 4 & 11 & 22 \\
\hline
\end{tabular}

Abbreviation: SNP, single nucleotide polymorphism.

aThe $P$-values were assessed by proper tests in each category. 


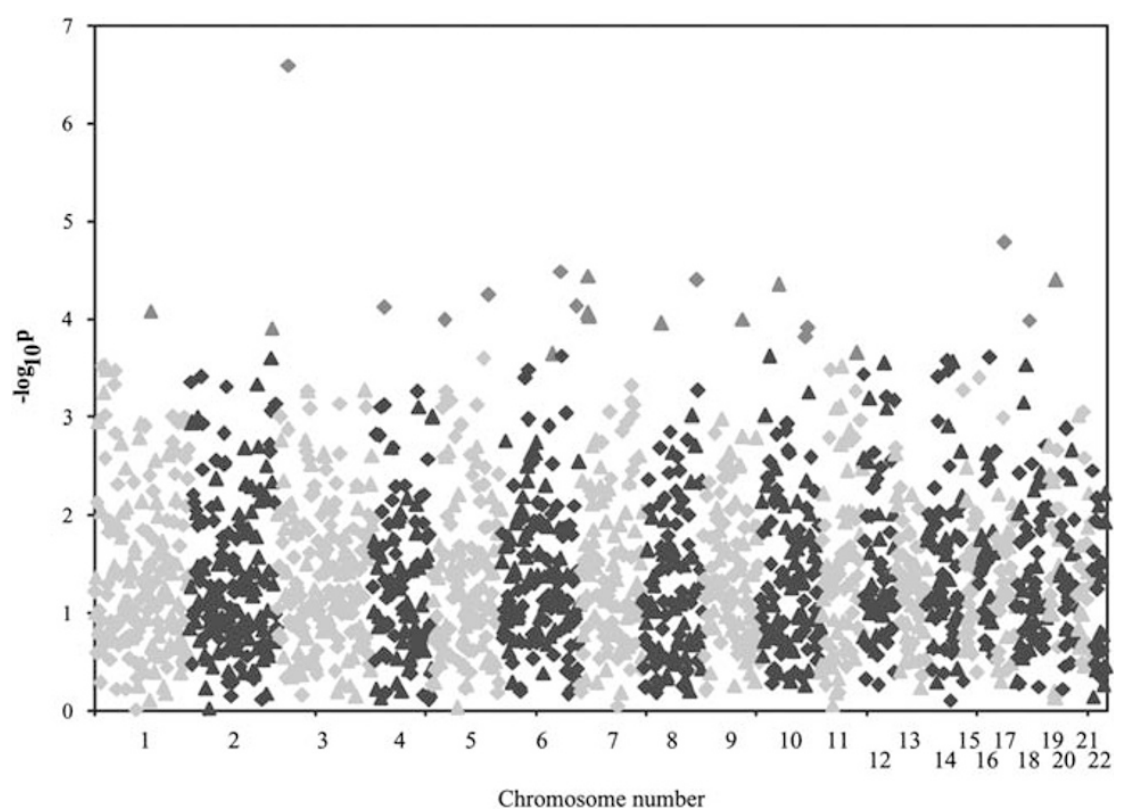

Figure 1 The scatter plot of $-\log _{10} P$ against all the analysis. The four data sets were plotted in $-\log _{10} P$ of GWAS. Triangles, diamonds and squares indicate SNPs in class $N, M$ and $C$, respectively.

stage. Following the quality control described in Materials and methods for samples and SNPs, we combined the data set of both stages. On the basis of the same criteria of classification as the first stage, association tests were performed for the 1803 SNPs that passed quality control (Figure 1 and Supplementary Table 1). Although none of the SNPs reached genome-wide significance level $(\alpha=0.05 /$ $250507 \approx 2 \times 10^{-7}$ ) after Bonferroni's correction, moderate associations were observed. We recruited the top 22 from 1833 SNPs, corresponding to $1.2 \%$ of filtered SNPs. The $P$-values of the 22 SNPs were $<0.0002$ (Table 2). Of the 22 SNPs, 7 (32\%) were selected from class M, 4 (18\%) from class $\mathrm{C}$ and 11 (50\%) from class $\mathrm{N}$.

\section{Intensified study}

We performed an intensified study for the 22 SNPs using larger casecontrol samples comprised of the GWAS cohort and additional samples to determine whether these SNPs would be noteworthy to be reported (Table 3). Specifically, they were genotyped in 1973 samples (904 controls/1069 cases). Following the quality control, we performed a statistical test for each SNP according to the classification at the GWA stages, and additionally calculated FPRPs. We finally identified five SNPs (rs7550260, rs7781293, rs4628172, rs1930095 and rs9864101) whose FPRP values with most stringent previous probability ( 1 in 10000 ) were $<0.5$. Of them, only rs1930095, on chromosomes 9 had FPRP under significance level $(\mathrm{FPRP}=0.2)$ $\left(P=1.31 \times 10^{-5}, \mathrm{FPRP}=0.17\right)$. It is an intergenic SNP and about $250 \mathrm{~kb}$ upstream from the nearest gene, actin-like $7 \mathrm{~B}$. Of the four SNPs with FPRPs under suggestive level $(F P R P=0.5)$, rs4628172 and rs7781293 $\left(P=1.32 \times 10^{-5}\right.$ and $2.78 \times 10^{-5}$, respectively) were in physical proximity to each other, and located on the transmembrane protein 195 gene (TMEM195) on chromosome 7. The SNP rs7550260 with a $P$-value of $4.93 \times 10^{-5}$ were located on Rho guanine nucleotideexchange factor 11 (ARHGEF11) on chromosome 1. The last one, rs9864101 existed within the intron of the IQ motif and the Sec7 domain 1 gene (IQSEC1), annotated by expressed sequence tag, on chromosome 3. Per-allele ORs of the Tallele of rs9864101 in male and female cohorts were 1.49 (95\% confidence interval (CI), 1.24-1.80;
$\left.P=3.63 \times 10^{-5}\right)$ and 1.07 (95\% CI, 0.89-1.29; $\left.P=0.46\right)$, respectively. The effect modification by gender was significant $(\psi$-statistic $=0.33$; $\left.\sigma^{2}=0.018 ; P=0.014\right)$, and thus the association of the SNP with IA was observed only in the male subjects. The $P$-values of the five SNPs described above were led by CA trend test, and the ORs increased linearly with the number of risk allele. Furthermore, for these five SNPs, we investigated whether the association results were affected by potential confounding factors, gender and hypertension. When performing a multivariate logistic regression with gender and hypertension as covariates, we still observed significant associations of the SNPs (data not shown).

In contrast, SNPs in class $\mathrm{C}$ and associated only in female subjects had high FPRP values and were weakly or not associated with IA.

\section{DISCUSSION}

In this study, we performed a two-stage genome-wide scan in Japanese cohorts, followed by an intensified study, through which we identified five promising SNPs that may be responsible for predisposition to IA. The two SNPs with the second and forth smallest $P$-value, rs4628172 $\left(P=1.32 \times 10^{-5}\right)$ and $\operatorname{rs7781293}\left(P=2.78 \times 10^{-5}\right)$ were located on TMEM195 on chromosome 7 . The $r^{2}$ linkage disequilibrium between the SNPs was 0.78 in our data set, and their physical distance was $4.1 \mathrm{~kb}$ in length. TMEM195 is a member of the fatty-acid hydroxylase family, which has transmembrane domains, although the function is not fully understood. The SNP rs7550260 was located on ARHGEF11 on chromosome 1 . The encoded protein may form a complex with $\mathrm{G}$ proteins and stimulate Rho-dependent signals. Intriguingly, in rat, overexpression of the ortholog induces the reorganization of the actin cytoskeleton and the formation of membrane ruffling. ${ }^{23}$ The SNP rs9864101, whose effect on IA was modified by gender, is located in the gene IQSEC1. ARF-GEP100 protein (ADP-ribosylation factorguanine nucleotide-exchange protein $-100 \mathrm{kDa}$ ) encoded by this gene activates ADP-ribosylation factor protein, ARF6, and regulates cell adhesion through recycling E-cadherin and remodeling actin cytoskeleton. ${ }^{24}$ We speculate that these three products might act in the uniform pathway of actin remodeling with $E L N / L I M K^{25}$ in smooth 


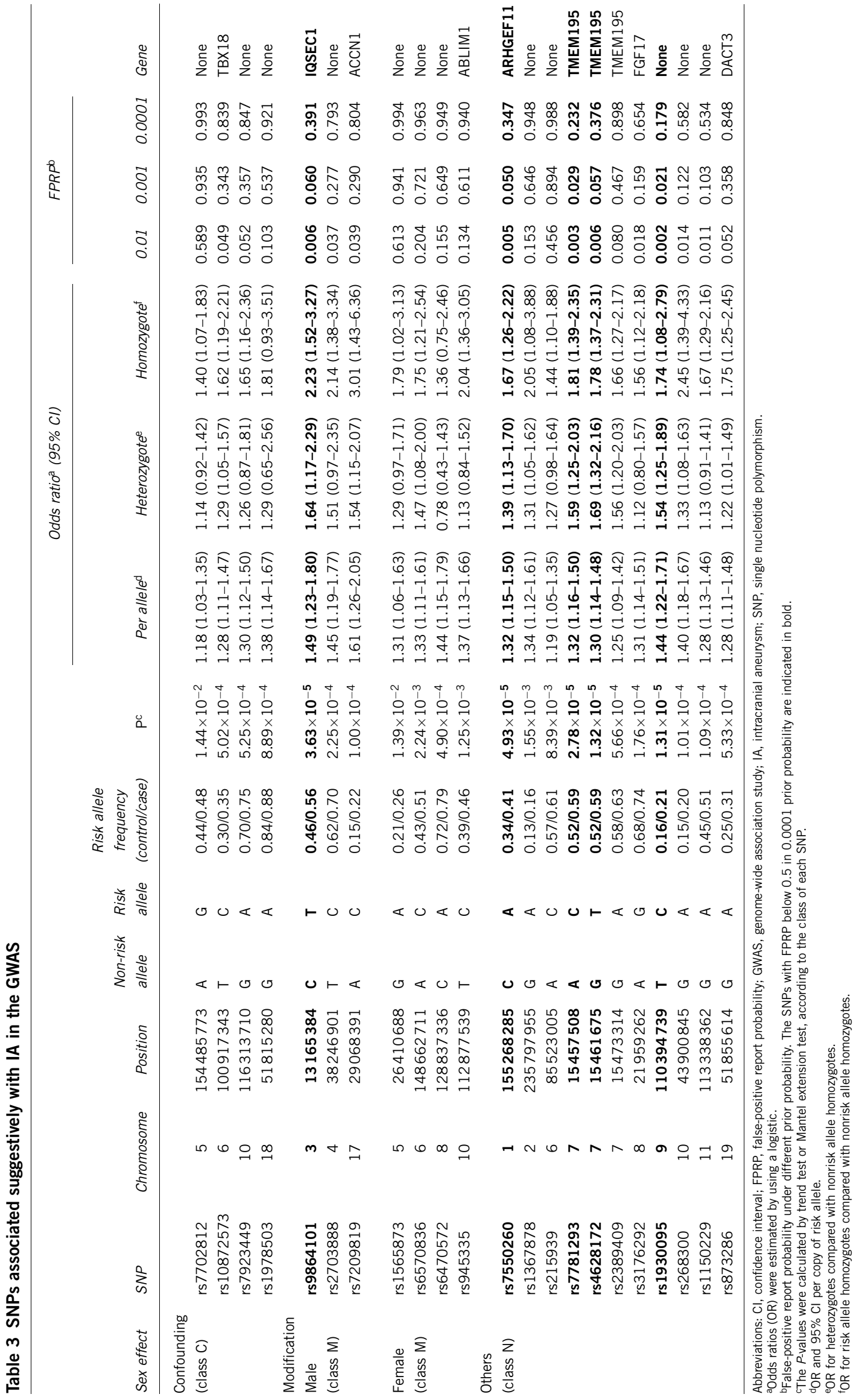


muscle cells in blood vessels, and that disturbance of these gene functions might cause IA formation through altering plasticity of the arterial wall.

Among the SNPs to which gender acts as an effect modifier or confounder, rs9864101 was the only associated SNP (with FPRP <0.5) whose genetic effect on IA was exerted in males only. Possibly, there was no association between IA and the SNPs in class M and C, except for rs9864101, but their risk allele frequencies or ORs could also have been too low to detect their genetic effects in this study design. In particular, for the SNPs in class M, the data set must be stratified by gender and then the sample size decreased by half. Thereby the statistical power is decreased.

We only focused on gender as a potential confounder or effect modifier of genetic association with IA in this study, because female gender is an established risk factor of IA. The gender is a distinct parameter, thus, it is filled almost entirely and accurately. On the other hand, the lifestyles, that is, smoking habit and frequent alcohol intake, of each individual are unstable over time and not completely described. We did not perform the GWA analysis after controlling for these important risk factors. Therefore, we may overlook SNPs modified and/or confounded by nongenetic factors other than gender. We also recognize the importance of the analysis taking lifestyle risk factors into account at a discovery stage, and thus the analysis is a challenge for the future.

Because Bilguvar et al. ${ }^{11}$ have replicated three SNPs significantly associated with IA by using a subset of our Japanese cohort (rs1429412 on chromosome 2q, rs10958409 on chromosome 8q and rs1333040 on chromosome 9p in 495 IA cases and 676 non-IA controls), the SNPs are likely to be true positives. In our study, we tested for association between the three SNPs and IA at the first stage of the current GWAS (194 IA cases and 288 controls) and obtained ORs and 95\% CIs for the SNPs as follows: rs1429412, $\mathrm{OR}=1.13 \quad(95 \% \mathrm{CI}, \quad 0.79-1.33)$; rs10958409, 1.20 (0.78-1.53); and rs1333040, 1.29 (0.98-1.72). The ORs were not so different from those in the previous study, ${ }^{11}$ but the effect sizes showed no statistical significance. This GWAS has limited power to detect loci with small effect sizes (for example, $<10 \%$ power to detect common variants with genotypic relative risk of 1.3 ; also see Supplementary Figure 1). We therefore think that this is the primary reason we could not detect the previously identified loci in this study. On the other hand, none of the SNPs identified in this study were associated with IA in Bilguvar et al.' s study (data not shown, personal communication from Professor M. Gunel, Yale University). Although we cannot rule out the possibility of the false-positive SNPs, our findings are suggested to be Japanese population-specific susceptibility variants, and the genes and genetic regions would be powerful candidates for IA-predisposing factors in the Japanese population. To prove this, it will be necessary to carry out more detailed investigations using larger sample sizes of independent sets of Japanese and/or Asian cohorts. We further provided the information of allele frequencies of 1833 SNPs filtered out through the current GWAS (Supplementary Table 1). We expect that this is helpful for future studies in search of Japanese- and/or Asian-specific susceptibility loci for IA.

\section{ACKNOWLEDGEMENTS}

We are grateful to the DNA donors and the supporting staffs for making this study possible. We thank Dr Hiroyuki Akagawa for his assistance with DNA specimen management. We also thank Makiko Funamizu, Midori Yamamoto, Kayako Fukuyama, Kozue Otaka, Eriko Tokubo, Hiromi Kamura and Miho Takabe for their technical help. This work was supported in part by CREST
(Core Research for Evolutionary Science and Technology) of Japan Science and Technology (II) and a Grant-in-Aid for scientific research on Priority Area 'Applied Genomics' from the Japanese Ministry of Education, Science, Sports and Culture (II).

1 Inagawa, T., Tokuda, Y., Ohbayashi, N., Takaya, M. \& Moritake, K. Study of aneurysmal subarachnoid hemorrhage in Izumo City, Japan. Stroke 26, 761-766 (1995).

2 Longstreth, W. T. Jr., Nelson, L. M., Koepsell, T. D. \& van Belle, G. Clinical course of spontaneous subarachnoid hemorrhage: a population-based study in King County, Washington. Neurology 43, 712-718 (1993).

3 Schievink, W. I., Wijdicks, E. F., Parisi, J. E., Piepgras, D. G. \& Whisnant, J. P. Sudden death from aneurysmal subarachnoid hemorrhage. Neurology 45, 871-874 (1995).

4 Onda, H., Kasuya, H., Yoneyama, T., Takakura, K., Hori, T., Takeda, J. et al. Genomewide-linkage and haplotype-association studies map intracranial aneurysm to chromosome 7q11. Am. J. Hum. Genet. 69, 804-819 (2001).

5 Akagawa, H., Tajima, A., Sakamoto, Y., Krischek, B., Yoneyama, T., Kasuya, H. et al. A haplotype spanning two genes, ELN and LIMK1, decreases their transcripts and confers susceptibility to intracranial aneurysms. Hum. Mol. Genet. 15, 1722-1734 (2006).

6 Akagawa, H., Narita, A., Yamada, H., Tajima, A., Krischek, B., Kasuya, H. et al. Systematic screening of lysyl oxidase-like (LOXL) family genes demonstrates that LOXL2 is a susceptibility gene to intracranial aneurysms. Hum. Genet. 121, 377-387 (2007).

7 Mineharu, Y., Inoue, K., Inoue, S., Kikuchi, K., Ohishi, H., Nozaki, K. et al. Association analyses confirming a susceptibility locus for intracranial aneurysm at chromosome 14q23. J. Hum. Genet. 53, 325-332 (2008).

8 Ruigrok, Y. M., Rinkel, G. J., van't Slot, R., Wolfs, M., Tang, S. \& Wijmenga, C. Evidence in favor of the contribution of genes involved in the maintenance of the extracellular matrix of the arterial wall to the development of intracranial aneurysms. Hum. Mol. Genet. 15, 3361-3368 (2006).

9 Ruigrok, Y. M., Rinkel, G. J. \& Wijmenga, C. The versican gene and the risk of intracranial aneurysms. Stroke 37, 2372-2374 (2006).

10 Weinsheimer, S., Goddard, K. A., Parrado, A. R., Lu, Q., Sinha, M., Lebedeva, E. R. et al. Association of kallikrein gene polymorphisms with intracranial aneurysms. Stroke 38, 2670-2676 (2007).

11 Bilguvar, K., Yasuno, K., Niemela, M., Ruigrok, Y. M., von Und Zu Fraunberg, M., van Duijn, C. M. et al. Susceptibility loci for intracranial aneurysm in European and Japanese populations. Nat. Genet. 40, 1472-1477 (2008).

12 WTCCC. Genome-wide association study of 14,000 cases of seven common diseases and 3,000 shared controls. Nature 447, 661-678 (2007).

13 Broderick, J. P., Viscoli, C. M., Brott, T., Kernan, W. N., Brass, L. M., Feldmann, E. et al. Major risk factors for aneurysmal subarachnoid hemorrhage in the young are modifiable. Stroke 34, 1375-1381 (2003).

14 Krischek, B. \& Inoue, I. The genetics of intracranial aneurysms. J. Hum. Genet. 51, 587-594 (2006).

15 Sankai, T., Iso, H., Shimamoto, T., Kitamura, A., Naito, Y., Sato, S. et al. Prospective study on alcohol intake and risk of subarachnoid hemorrhage among Japanese men and women. Alcohol. Clin. Exp. Res. 24, 386-389 (2000).

16 Yamada, S., Koizumi, A., Iso, H., Wada, Y., Watanabe, Y., Date, C. et al. Risk factors for fatal subarachnoid hemorrhage: the Japan Collaborative Cohort Study. Stroke 34, 2781-2787 (2003).

17 Skol, A. D., Scott, L. J., Abecasis, G. R. \& Boehnke, M. Joint analysis is more efficient than replication-based analysis for two-stage genome-wide association studies. Nat. Genet. 38, 209-213 (2006).

18 Purcell, S., Neale, B., Todd-Brown, K., Thomas, L., Ferreira, M. A., Bender, D. et al. PLINK: a tool set for whole-genome association and population-based linkage analyses. Am. J. Hum. Genet. 81, 559-575 (2007).

19 Thomas, D. C. Statistical Methods in Genetic Epidemiology (Oxford University, New York, 2004).

20 Wacholder, S., Chanock, S., Garcia-Closas, M., El Ghormli, L. \& Rothman, N. Assessing the probability that a positive report is false: an approach for molecular epidemiology studies. J. Natl Cancer Inst. 96, 434-442 (2004).

21 Chanock, S. Candidate genes and single nucleotide polymorphisms (SNPs) in the study of human disease. Dis. Markers 17, 89-98 (2001).

22 Samani, N. J., Erdmann, J., Hall, A. S., Hengstenberg, C., Mangino, M., Mayer, B. et al. Genomewide association analysis of coronary artery disease. N. Engl. J. Med. 357, 443-453 (2007).

23 Jackson, M., Song, W., Liu, M. Y., Jin, L., Dykes-Hoberg, M., Lin, C. I. et al. Modulation of the neuronal glutamate transporter EAAT4 by two interacting proteins. Nature 410, 89-93 (2001).

24 Hiroi, T., Someya, A., Thompson, W., Moss, J. \& Vaughan, M. GEP100/BRAG2: activator of ADP-ribosylation factor 6 for regulation of cell adhesion and actin cytoskeleton via E-cadherin and alpha-catenin. Proc. Natl Acad. Sci. USA 103, 10672-10677 (2006)

25 Maekawa, M., Ishizaki, T., Boku, S., Watanabe, N., Fujita, A., Iwamatsu, A. et al. Signaling from Rho to the actin cytoskeleton through protein kinases ROCK and LIMkinase. Science 285, 895-898 (1999). 